\title{
Prevalência de doenças crônicas auto-referidas e utilização de serviços de saúde, PNAD/1998, Brasil
}

\author{
Prevalence of self reported chronic diseases and \\ health services consumption from the National \\ Household Sample Survey of 1998 in Brazil
}

Márcia Furquim de Almeida 1

Rita Barradas Barata 2

Cláudia Valencia Montero 3

Zilda Pereira da Silva 4

1 Departamento de Epidemiologia da Faculdade de Saúde Pública da USP. Av. Dr. Arnaldo 715, 01246-904 São Paulo SP. marfural@usp.br

2 Departamento de Medicina Social da Faculdade de Ciências Médicas da Santa Casa de São Paulo.

3 Centro de Vigilância Epidemiológica Prof. Alexandre Vranjac/SES/SP.

4 Fundação SEADE.
Abstract The prevalence and the distribution of chronic diseases became of overwhelming importance with the ageing process of Brazilian population. These diseases are responsible by an increased demand of health services. It was analyzed the data from the sample of the Health Supplement of PNAD/98 (National Household Sample Survey). It was used the data from all self reported chronic diseases by sociodemographic variables and health services consumption. It was employed prevalence ratios and odds ratios, with $95 \%$ confidence interval to evaluate the presence of possible associations. As expected it was observed an increase of chronic diseases prevalence with age; and a decrease with educational and income level. It was found higher rates of chronic diseases in women and in the group without access to private health insurance. Consumption of health services was 1.8 fold for the chronic diseases group. It was not found an association between the mean number of medical visits and income level, although it was observed a clear differential in hospitalization rates, with higher rates of hospitalization in the higher income brackets. It was not also found differences of the mean number of medical visits between the owners of private health insurance and the users of the Brazilian National Health System (SUS).

Key words Self reported chronic diseases, Prevalence, Sociodemographic differentials, Health services consumption
Resumo Com o envelhecimento da população brasileira torna-se cada vez mais importante conhecer a prevalência das doenças crônicas. Essas doenças constituem-se em forte demanda aos serviços de saúde. Foram utilizados os dados da amostra da PNAD/98. Analisou-se o conjunto de doenças crônicas auto-referidas, empregou-se a razão de prevalência e razão de odds ratios com intervalo de confiança de $95 \%$ para verificar a presença de associações. Comprovou-se o aumento da prevalência das doenças crônicas com o aumento da idade; padronizando-se a idade identificou-se um gradiente de redução da prevalência com aumento da escolaridade e da renda. Observou-se maior prevalência entre mulheres e entre os que não possuíam plano de saúde. A presença de doença crônica estava associada à má avaliação do estado de saúde e de restrição de atividade. A utilização dos serviços de saúde foi de 1,8 vezes entre os portadores de doenças crônicas; com um consumo significativamente maior do número médio de consultas. Não se verificou diferença significante do número médio de consultas médicas por estrato de renda. Entre os portadores de doença crônica não houve diferença significativa do número médio de consultas entre usuários do SUS e de planos privados de saúde.

Palavras-chave Doença crônica auto-referida, Prevalência, Diferenciais socioeconômicos, Utilização de serviços de saúde 
As doenças crônicas são a principal causa de incapacidade, a maior razão para a demanda a serviços de saúde e respondem por parte considerável dos gastos efetuados no setor. Segundo Lorig et al. (2001) a prevalência de problemas crônicos de saúde vem aumentando, entre os adultos, em todos os grupos etários. Elliot et al. (2000) referem que $47 \%$ da população do Reino Unido relata dor crônica em diferentes localizações. Estudando idosos de uma comunidade em Porto Rico, Cordero et al. (2000) registram $20,0 \%$ de prevalência de hipertensão arterial, $17,8 \%$ de artrites e reumatismos, $16,5 \%$ de doenças do coração e 10,9\% de diabetes.

Diferentemente de outros problemas de saúde, os problemas crônicos parecem afetar com freqüência semelhante os diversos grupos sociais, ainda que sua gravidade possa variar, sendo maior entre os socialmente excluídos, conforme observado por Trevena et al. (2001) ao comparar a prevalência de doenças crônicas entre moradores de rua e a população geral em Sidney, Austrália.

A utilização de serviços de saúde pelos portadores de problemas crônicos de saúde é consideravelmente maior do que a observada entre a população em geral. Analisando os gastos em saúde efetuados pelas organizações administradoras de saúde (HMO) norte-americanas, Chris Rauber (1999) aponta uma estrutura de gastos que destina $84 \%$ dos recursos para tratamento e recuperação e apenas $16 \%$ para prevenção dos agravos. Os portadores de doenças crônicas, embora correspondam a cerca de $20 \%$ dos clientes, consomem cerca de $80 \%$ dos recursos.

A procura por serviços de saúde e sua utilização dependem de uma série de fatores que podem ser agrupados em três categorias - predisposição, capacidade e necessidade -, conforme o esquema utilizado por Guerra et al. (2001) no estudo de uma coorte de idosos em Bambuí (MG). Entre as características que predispõem à utilização de serviços de saúde encontram-se variáveis sociodemográficas como idade, gênero, raça, hábitos, etc. A capacidade de consumo de serviços é condicionada pela renda, cobertura securitária pública ou privada, e pela oferta de serviços, entre outras características. Finalmente, a necessidade pode ser traduzida na existência de diagnósticos prévios, autopercepção de problemas de saúde ou de estado de saúde insatisfatório (demanda espontânea), ou ainda, ser induzida pela detecção técnica de problemas que não eram sentidos co- mo tais pelos próprios indivíduos (demanda induzida) (Branch, 2000).

Assim, a presença de um problema crônico de saúde, seja o seu conhecimento pelo indivíduo resultante de um diagnóstico médico anterior, seja derivado de autopercepção, constitui um dos determinantes proximais mais fortes para a procura e utilização de serviços de saúde.

O suplemento de saúde da PNAD/98, aplicado a uma amostra populacional com representação nacional, possibilita a estimativa da prevalência de problemas crônicos de saúde e permite também estudar as características sociodemográficas desses indivíduos portadores de problemas crônicos, bem como seu perfil de uso dos serviços de saúde, em comparação com o restante da população.

Diversos estudos têm sido feitos no sentido de validar as informações de morbidade ou estado de saúde auto-referidos, visando fortalecer as análises e interpretações obtidas em inquéritos domiciliares. Rathouz et al. (1998) estudaram a consistência de informações auto-referidas sobre capacidade física em idosos, mostrando que ela se mantém alta durante um seguimento de 24 semanas. Ferraro e Su (2000) investigaram qual das duas fontes de informação - exames clínicos ou auto-avaliação - eram mais úteis para fins prognósticos, concluindo que ambas as medidas são preditoras de incapacidade, em um período de 10 anos, mas as associações mais fortes foram observadas para a morbidade auto-referida, principalmente para doenças crônicas não severas. Benyamini et al. (2000) referem que os indivíduos que avaliam seu estado de saúde como regular ou ruim tem de 2 a 5 vezes mais risco de morrer, dentro de 2 a 13 anos, do que aqueles que avaliam sua saúde como boa ou muito boa.

Wu et al. (2000) estudaram a acurácia da prevalência auto-referida de doenças crônicas comparada aos diagnósticos clínicos, entre idosos de Taiwan, observando que a morbidade auto-referida tendeu a subestimar de maneira importante (21\% a menos) a prevalência de doenças cardíacas; levemente ( $4 \%$ a menos) a prevalência de hipertensão arterial; e a superestimar ligeiramente ( $2 \%$ a mais) a prevalência de diabetes. Enns et al. (2000) obtiveram correlação significativa $(r=0,40 ; p<0,01)$ entre depressão auto-referida e observada entre adultos não psicóticos não usuários de drogas. Cox et al. (2000) encontraram correlação significante $(r=0,32 ; p<0,005)$ entre lombalgia au- 
to-referida e escala padronizada de performance embora nenhuma correlação fosse observada entre lombalgia e a escala de dor $(r=-0,17$; $\mathrm{p}=0,13)$.

$\mathrm{Na}$ PNAD/98 a informação sobre a presença de doença crônica foi obtida através de respostas - sim ou não - para 12 condições ou diagnósticos. Tendo em vista que a prevalência auto-referida de doenças crônicas como um todo parece ter, em estudos semelhantes, maior acurácia do que a prevalência auto-referida de problemas específicos de saúde, optou-se por analisar, neste artigo, a prevalência de doenças crônicas em conjunto.

A pesquisa procurou responder a três ordens de questões:

- a informação de doença crônica auto-referida é consistente com a auto-avaliação do estado de saúde, restrição das atividades cotidianas por motivo de saúde e haver estado acamado? - quais são as características sociodemográficas dos indivíduos que referiram doenças crônicas e em que medida elas diferem das características daqueles que não referiram essas doenças?

- qual é o perfil de uso de serviços de saúde desse grupo de pessoas?

\section{Metodologia}

Doença crônica foi definida na PNAD como uma afecção de saúde que acompanha o indivíduo por longo período de tempo, podendo apresentar momentos de piora ou melhora sensível (episódios agudos). Foram considerados portadores de doenças crônicas aqueles indivíduos que responderam afirmativamente à presença de pelo menos um dos seguintes problemas de saúde: doença da coluna ou das costas, artrite ou reumatismo, câncer, diabetes, bronquite ou asma, doença do coração, hipertensão, doença renal crônica, depressão, tuberculose, tendinite ou tenossinovite e cirrose.

Para efeito desta publicação foram utilizados os dados obtidos na amostra, sem expandilos para a população, a fim de poder calcular as medidas de risco relativo (razão de prevalências e odds ratio) e intervalos de confiança, comparando os indivíduos portadores e não de problemas crônicos, ou os diferentes estratos entre os portadores de doenças crônicas.

Os indivíduos com menos de 15 anos de idade foram excluídos da população de interesse, tendo em vista a pequena incidência de problemas crônicos antes dessa idade. Assim, as informações analisadas referem-se a 240.821 pessoas entrevistadas, com idade de 15 anos e mais.

Para a validação interna da informação sobre a presença de doença crônica foram utilizadas três variáveis:

- a auto-avaliação do estado de saúde expressa segundo um dos cinco estados: muito bom, bom, regular, ruim e muito ruim;

- a restrição das atividades cotidianas por motivo de saúde definida como a impossibilidade de realizar as tarefas habituais durante pelo menos um dia nos 15 dias que antecederam a entrevista;

- ter ou não estado acamado por motivo de saúde, em casa ou no hospital, durante pelo menos meio dia nos 15 dias que antecederam a entrevista.

Os motivos para a restrição das atividades também foram investigados para verificar se havia coerência entre referir doenças crônicas e apresentar restrições compatíveis com elas. Os motivos referidos foram agrupados em problemas respiratórios; problemas do coração ou pressão arterial; dor nos braços ou mãos; problema mental ou emocional; outras doenças; problemas odontológicos; causas externas compreendendo os acidentes de trabalho, de trânsito e outras agressões; e outros motivos.

Para a caracterização dos portadores de doença crônica foram selecionadas algumas variáveis sociodemográficas:

- sexo: masculino e feminino;

- idade agrupada nas faixas de 15 a 24 anos (jovens); 25 a 44 anos (adultos jovens); 45-64 anos (adultos) e 65 anos e mais (idosos);

- residência urbana ou rural;

- raça/cor branca ou negra, com as demais agrupadas no total;

- número de anos de escolaridade agrupados nas faixas de: até 3 anos (analfabetos funcionais); 4 a 7 anos (ensino fundamental incompleto); 8 a 10 anos (ensino médio incompleto); 11 anos e mais (ensino médio e/ou superior);

Tendo em vista que a variável idade está fortemente associada à prevalência de doenças crônicas, optou-se por ajustar todas as prevalências por idade antes de proceder às comparações. Portanto, todas as taxas de prevalência são apresentadas após o ajuste por idade e expressas em casos por 100 habitantes, uma vez que a ocorrência de problemas crônicos de saúde é bastante freqüente na população $(41,5 \%$ dos entrevistados com idade de 15 anos e mais). 
Para avaliar a capacidade de utilização dos serviços de saúde pelos portadores de doença crônica foram selecionadas duas variáveis:

- renda familiar expressa em salários-mínimos e agrupada nas faixas de até um saláriomínimo (s. m.); mais de 1 a 2 s.m.; mais de 2 a 3 s.m.; mais de 3 a 5 s.m.; mais de 5 a 10 s.m.; mais de 10 a 20 s.m. e mais de 20 s.m.;

- possuir ou não um plano de saúde particular, empresarial ou de órgão público.

A utilização de serviços de saúde foi analisada por meio de um conjunto de informações incluindo o uso de consultas médicas ou internações hospitalares nos últimos 12 meses (excluídas as internações por parto e as que tiveram permanência inferior a 24 horas) e a procura ou não de serviços de saúde nos 15 dias que antecederam a entrevista. Para os indivíduos, portadores de doenças crônicas, que referiram um problema de saúde nos últimos 15 dias foram investigados:

- procura por serviços de saúde: sim ou não;

- atendimento realizado: sim ou não;

- $\quad$ atendimento pelo SUS: sim ou não;

- motivos para a procura agrupados em doença, prevenção, tratamento ou reabilitação e acidentes ou lesões;

- tipo de serviço procurado agrupado em serviços de atendimento ambulatorial, urgência ou emergência, farmácia, laboratório ou exames complementares, atendimento domiciliar;

- motivos para não atendimento;

- motivos para não procura.

Maiores detalhes sobre a amostra, o instrumento de coleta e as variáveis da PNAD podem ser obtidos na publicação do IBGE.

\section{Validação interna}

A utilização de dados relativos à morbidade referida tem sido bastante discutida no contexto dos inquéritos domiciliares como já mencionado na introdução deste artigo. Em recente editorial do British Medical Journal, Amartya Sen (2002), o ganhador do prêmio Nobel de economia, alerta para a questão dos equívocos que o uso de informações exclusivamente baseadas na percepção dos indivíduos pode produzir na avaliação das necessidades de cuidados médicos. Utilizando dados de províncias indianas e dos Estados Unidos, o autor discute as limitações da autopercepção dos problemas de saúde em função da experiência social dos indivíduos, mostrando que nas áreas onde há mais e melhores serviços de saúde e a população tem maior nível de escolaridade as pessoas têm probabilidades maiores de perceber suas doenças.

Tendo em conta essas e outras limitações da morbidade referida, considerou-se necessário validar internamente a informação da presença de doença crônica através da comparação da prevalência com outras informações relativas ao estado de saúde.

A tabela 1 apresenta a prevalência de problemas crônicos de saúde segundo a auto-avaliação do estado de saúde, restrição das atividades cotidianas e principais motivos, e ter estado acamado por motivo de saúde.

Há um nítido gradiente, inversamente proporcional, entre o estado de saúde e a prevalência de doenças crônicas. As razões de prevalências mostram que as referências a problemas crônicos de saúde foram 2,69 vezes mais freqüentes entre indivíduos que avaliaram seu estado de saúde como regular, e 3,15 vezes mais freqüentes entre as pessoas que classificaram seu estado de saúde como ruim ou muito ruim. Portanto, há coerência entre as duas avaliações.

Entre os portadores de problemas crônicos, a restrição das atividades cotidianas nos 15 dias que antecederam a entrevista foi 1,96 vezes mais freqüente do que a não restrição. Novamente, observa-se coerência entre as informações. Analisando os motivos de restrição, chama a atenção o fato de que o único motivo não associado com a presença de doença crônica foi a ocorrência de problema odontológico. Os demais motivos estão todos fortemente associados com a própria definição de problema crônico e, conforme o esperado, apresentam razões de prevalência altas. Para as causas externas, embora haja um excesso relativo de menções entre os portadores de problemas crônicos de saúde provavelmente relacionado à ocorrência de quedas entre idosos, a freqüência tende a se aproximar da observada para os indivíduos que não mencionaram problemas crônicos de saúde. Finalmente, verificou-se ainda que foi 1,95 vezes mais freqüente, para portadores de problemas crônicos, ter estado acamado por motivo de saúde nos 15 dias que antecederam a entrevista.

Portanto, todos os dados apresentam consistência sugerindo que, a despeito das restrições normalmente feitas à utilização de morbidade referida, a menção a problema crônico de saúde pode ser valorizada como um dos determinantes proximais na avaliação do uso de serviços de saúde. Mesmo considerando o ní- 
Tabela 1

Prevalência de doenças crônicas em pessoas com 15 anos e mais segundo auto-avaliação de saúde e restrição de atividades, Brasil, 1998.

\begin{tabular}{|c|c|c|c|c|c|c|c|c|}
\hline \multirow[t]{3}{*}{ Variáveis } & \multicolumn{6}{|c|}{ Declaração de Doença Crônica } & \multirow{3}{*}{$\begin{array}{l}\text { Razão de } \\
\text { Prevalência }\end{array}$} & \multirow[t]{3}{*}{ IC-95\% } \\
\hline & \multicolumn{2}{|c|}{ Sim } & \multicolumn{2}{|c|}{ Não } & \multicolumn{2}{|c|}{ Total } & & \\
\hline & $\mathrm{n}$ & taxa & $\mathrm{n}$ & taxa & $\mathrm{n}$ & taxa & & \\
\hline TOTAL & 99,963 & 41.51 & 140,858 & 58.49 & 240,821 & 100.00 & & \\
\hline \multicolumn{9}{|l|}{ Auto-avaliação do estado de saúde } \\
\hline Ruim e muito ruim & 10,503 & 88.69 & 1,340 & 11.31 & 11,843 & 100.00 & 3.15 & $3,12-3,18$ \\
\hline Regular & 39,823 & 75.70 & 12,780 & 24.30 & 52,603 & 100.00 & 2.69 & $2,67-2,71$ \\
\hline Muito bom e bom & 49,622 & 28.14 & 126,709 & 71.86 & 176,331 & 100.00 & 1.00 & \\
\hline \multicolumn{9}{|l|}{$\begin{array}{l}\text { Restrição de atividades } \\
\text { nas } 2 \text { últimas semanas }\end{array}$} \\
\hline $\operatorname{Sim}$ & 12,785 & 76.29 & 3,973 & 23.71 & 16,758 & 100.00 & 1.96 & $1,94-1,98$ \\
\hline Não & 87,178 & 38.91 & 136,885 & 61.09 & 224,063 & 100.00 & 1.00 & \\
\hline \multicolumn{9}{|l|}{ Esteve acamado } \\
\hline Sim & 8,148 & 77.64 & 2,347 & 22.36 & 10,495 & 100.00 & 1.95 & $1,93-1,97$ \\
\hline Não & 91,814 & 39.86 & 138,511 & 60.14 & 230,325 & 100.00 & 1.00 & \\
\hline \multicolumn{9}{|l|}{ Motivo da restrição } \\
\hline Problema respiratório & 1,127 & 76.98 & 337 & 23.02 & 1,464 & 100.00 & 1.86 & $1,81-1,92$ \\
\hline Problema de coração ou pressão & 2,130 & 98.29 & 37 & 1.71 & 2,167 & 100.00 & 2.40 & $2,38-2,42$ \\
\hline Dor nos braços ou nas mãos & 755 & 88.72 & 96 & 11.28 & 851 & 100.00 & 2.15 & $2,10-2,20$ \\
\hline Problema mental ou emocional & 778 & 81.30 & 179 & 18.70 & 957 & 100.00 & 1.97 & $1,91-2,07$ \\
\hline Outra doença & 6,070 & 73.87 & 2,147 & 26.13 & 8,217 & 100.00 & 1.83 & $1,80-1,86$ \\
\hline Problema odontológico & 134 & 49.45 & 137 & 50.55 & 271 & 100.00 & 1.19 & $1,06-1,34$ \\
\hline Causa externa & 593 & 57.91 & 431 & 42.09 & 1,024 & 100.00 & 1.40 & $1,33-1,47$ \\
\hline Outro motivo & 1,116 & 66.04 & 574 & 33.96 & 1,690 & 100.00 & 1.60 & $1,54-1,65$ \\
\hline
\end{tabular}

Fonte : Fundação IBGE. Pesquisa Nacional por Amostra de Domicílios - PNAD. Nota: Excluem os casos de resposta ignorada.

vel insatisfatório de escolaridade da população brasileira como um possível fator que levasse a erros na aferição dos problemas de saúde, os dados poderiam estar subestimados e não superestimados (Sen, 2002 e Wu et al., 2000).

\section{Características sociodemográficas dos portadores de problemas crônicos de saúde}

A tabela 2 apresenta as taxas de prevalência de doenças crônicas, ajustadas por idade, para algumas características sociodemográficas dos portadores. A prevalência variou, para os grupos etários estudados, entre 17,7 por 100 entrevistados no grupo de 15 a 24 anos, e 80,8 por 100 entrevistados no grupo de 65 anos e mais. Adotando como categoria de referência o grupo de jovens de 15 a 24 anos, observa-se que o risco de apresentar doenças crônicas foi 2,12 vezes maior para os adultos jovens de 25 a 44 anos; 3,71 vezes maior para os adultos de 45 a 64 anos; e 4,57 vezes maior para os idosos, conforme o esperado.

Em relação ao sexo, observou-se risco $25 \%$ maior para as mulheres. Tendo em vista que as mulheres tendem a informar, mais do que os homens, a presença de problemas de saúde, inclusive os de menor gravidade, tal diferença pode ser devida apenas à freqüência maior de menções a problemas crônicos entre as mulheres (Benyamini et al.; 2000; Enns et al.; 2000; Cox et al., 2000).

Embora os dados apontem prevalência ligeiramente mais alta entre os negros e a diferença seja estatisticamente significante, a razão de prevalência tem valor muito próximo de 1 , sugerindo que a diferença não é relevante.

Em relação ao nível de escolaridade, observou-se que o risco foi inversamente proporcional ao número de anos de escolaridade formal. Os indivíduos com menos de 3 anos de escolaridade apresentaram $28 \%$ mais casos do 
Tabela 2

Prevalência de doenças crônicas ajustadas por idade em pessoas de 15 anos e mais, segundo características sociodemográficas, Brasil, 1998.

\begin{tabular}{|c|c|c|c|c|c|c|}
\hline \multirow{2}{*}{$\begin{array}{l}\text { Características } \\
\text { Sociodemográficas }\end{array}$} & \multicolumn{2}{|c|}{ Doença crônica } & \multirow[t]{2}{*}{ Total } & \multirow{2}{*}{$\begin{array}{l}\text { Prevalência } \\
\text { Ajustada }\end{array}$} & \multirow{2}{*}{$\begin{array}{l}\text { Razão de } \\
\text { Prevalência }\end{array}$} & \multirow[t]{2}{*}{ IC - 95\% } \\
\hline & $\mathrm{n}$ & Prevalência & & & & \\
\hline \multicolumn{7}{|l|}{ Grupos de idade } \\
\hline de 15 a 24 anos & 12,084 & 17.7 & 68,346 & & 1.00 & \\
\hline de 25 a 44 anos & 37,674 & 37.5 & 100,556 & & 2.12 & $2,08-2,16$ \\
\hline de 45 a 64 anos & 34,250 & 65.7 & 52,159 & & 3.71 & $3,65-3,78$ \\
\hline de 65 anos e mais & 15,944 & 80.8 & 19,727 & & 4.57 & $4,49-4,65$ \\
\hline \multicolumn{7}{|l|}{ Sexo } \\
\hline homens & 41,576 & 36.0 & 115,361 & 36.8 & 1.00 & \\
\hline mulheres & 58,387 & 46.5 & 125,460 & 46.0 & 1.25 & $1,24-1,26$ \\
\hline \multicolumn{7}{|l|}{ Raça } \\
\hline branca & 52,684 & 41.7 & 126,263 & 40.5 & 1.00 & \\
\hline negra (preto + pardo) & 46,619 & 41.3 & 112,874 & 42.9 & 1.06 & $1,05-1,07$ \\
\hline \multicolumn{7}{|l|}{ Escolaridade } \\
\hline até 3 Anos & 39,652 & 55.9 & 70,965 & 45.8 & 1.28 & $1,26-1,29$ \\
\hline de 4 a 7 anos & 30,157 & 39.0 & 77,292 & 42.0 & 1.17 & $1,15-1,19$ \\
\hline de 8 a 10 anos & 12,247 & 30.7 & 39,834 & 39.1 & 1.09 & $1,07-1,11$ \\
\hline de 11 anos e mais & 17,529 & 34.0 & 51,510 & 35.9 & 1.00 & \\
\hline \multicolumn{7}{|l|}{ Situação } \\
\hline urbana & 82,179 & 41.1 & 199,936 & 41.3 & 1.00 & \\
\hline rural & 17,784 & 43.5 & 40,885 & 42.9 & 1.04 & $1,03-1,05$ \\
\hline \multicolumn{7}{|l|}{ Renda familiar } \\
\hline sem rendimentos & 1,939 & 35.4 & 5,478 & 43.9 & 1.30 & $1,25-1,35$ \\
\hline até 1 S.M. & 9,830 & 49.3 & 19,923 & 47.6 & 1.41 & $1,37-1,44$ \\
\hline mais de 1 a 2 S.M. & 15,841 & 45.6 & 34,745 & 45.3 & 1.34 & $1,31-1,37$ \\
\hline mais de 2 a 3 S.M. & 13,502 & 44.3 & 30,457 & 44.1 & 1.30 & $1,27-1,34$ \\
\hline mais de 3 a 5 S.M. & 19,050 & 41.0 & 46,429 & 42.1 & 1.25 & $1,22-1,27$ \\
\hline mais de 5 a 10 S.M. & 19,723 & 39.6 & 49,782 & 40.2 & 1.19 & $1,16-1,22$ \\
\hline mais de 10 a 20 S.M. & 10,523 & 37.6 & 27,985 & 37.2 & 1.10 & $1,07-1,13$ \\
\hline mais de 20 S.M. & 6,331 & 35.5 & 17,857 & 33.8 & 1.00 & \\
\hline \multicolumn{7}{|l|}{ Plano de saúde } \\
\hline Sim & 26,182 & 41.6 & 62,983 & 40.0 & 1.00 & \\
\hline Não & 73,779 & 41.5 & 177,832 & 42.2 & 1.06 & $1,05-1,07$ \\
\hline
\end{tabular}

Fonte: Fundação IBGE. Pesquisa Nacional por Amostra de Domicílios - PNAD.

Nota: Excluem os casos de resposta ignorada.

que aqueles com 11 anos ou mais de escolaridade. A diferença foi de $17 \%$ entre aqueles com 4 a 7 anos de escolaridade e caiu para $9 \%$ entre aqueles com 8 a 10 anos de escolaridade.

Mesmo considerando-se que o acesso a serviços de saúde e o nível de escolaridade possam exercer um papel importante na percepção dos indivíduos para as doenças crônicas, no caso brasileiro observou-se gradiente inverso entre a prevalência e a escolaridade, à exceção do grupo de jovens onde praticamente não há diferenças para os diversos níveis de escolaridade (Figura 1).
Quanto à residência em zona urbana ou rural, a diferença de prevalência, embora estatisticamente significante, não foi suficiente para indicar uma diferença real de risco. É importante lembrar que a amostra da PNAD não inclui a zona rural da região Norte, isto é, cerca de 4 milhões (12,2\%) dos 32 milhões de brasileiros que ainda vivem na zona rural. Como esses indivíduos correspondem a apenas $2,8 \%$ da população brasileira, talvez sua exclusão não modifique substancialmente os resultados.

A prevalência de problemas crônicos foi inversamente proporcional à renda familiar, com 
Figura 1

Traxa de prevalência de doenças crônicas segundo escolariddade e faixa etária, Brasil, 1998.

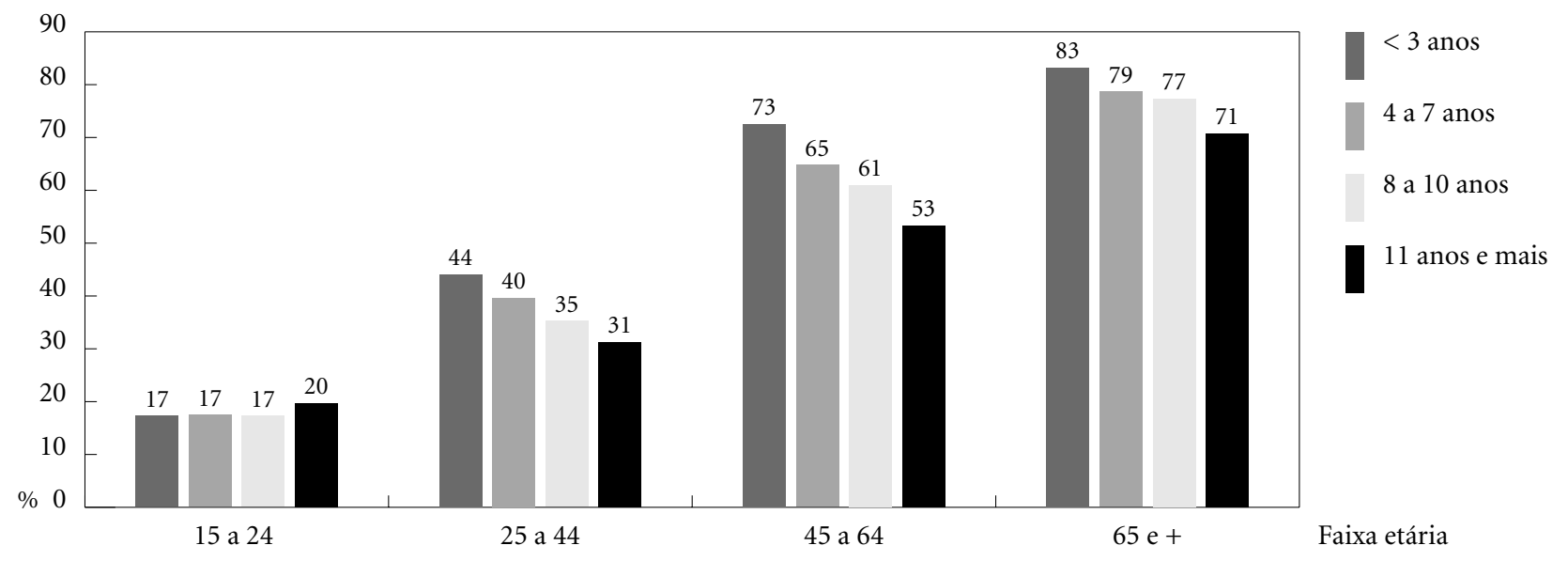

exceção do grupo de indivíduos sem rendimentos, para os quais a taxa foi um pouco menor do que a observada nos dois grupos subseqüentes. Tal resultado deve ser avaliado com cautela tendo em vista a possibilidade de subinformação para os indivíduos submetidos a piores condições de privação social (Trevena et al., 2001; Sen, 2002). Além disso, número reduzido de participantes na amostra $(2,2 \%)$ pertence à categoria dos sem rendimentos. Para as demais faixas de renda observou-se risco decrescente: os indivíduos que tinham até um salário-mínimo de renda familiar mensal apresentaram prevalência de problemas crônicos $41 \%$ maior do que aqueles cuja renda familiar mensal era maior do que 20 salários-mínimos.

Portanto, se o nível de escolaridade e a renda familiar mensal fossem tomados como indicadores de condições sociais, os dados permitiriam afirmar que a prevalência de problemas crônicos de saúde, embora extremamente freqüente em todos os grupos sociais, apresentou risco distinto nos diferentes estratos sociais.

Finalmente, a prevalência entre os possuidores de planos de saúde - particular, empresarial, ou de órgão público - foi semelhante àquela observada entre os não possuidores. Ainda que a diferença observada seja estatisticamente significante, ela não apresenta relevância. Tal resultado deve-se ao fato de parte significativa dos planos ser adquirida em decor- rência da inserção profissional, através de planos para empresas, não correspondendo diretamente a simples posse a melhor ou pior condição social.

\section{Utilização de serviços de saúde pelos portadores de problemas crônicos de saúde}

Como já mencionado na introdução deste artigo, o consumo de serviços de saúde depende de um conjunto de variáveis que podem ser agrupadas em termos de características do usuário, capacidade de consumo avaliada pela oferta de serviços e pela renda e/ou cobertura através de sistemas de seguro públicos ou privados e necessidade definida pela presença de um problema de saúde percebido como tal (Guerra et al., 2001).

Neste artigo procuramos comparar o perfil de utilização de serviços de saúde dos indivíduos portadores de problemas crônicos de saúde e daqueles que não mencionaram esse tipo de problema, na população brasileira com 15 anos ou mais.

A tabela 3 apresenta as informações relativas ao uso de serviços de saúde para os dois grupos, com e sem problemas crônicos de saúde, e a comparação, através da odds ratio, do padrão de utilização. 
Tabela 3

Consumo de serviços de saúde segundo presença de doenças crônicas, Brasil 1998.

\begin{tabular}{|c|c|c|c|c|c|c|c|c|}
\hline \multirow[t]{3}{*}{ Variáveis } & \multicolumn{6}{|c|}{ Declaração de Doença Crônica } & \multirow{3}{*}{$\begin{array}{l}\text { Odds } \\
\text { Ratio }\end{array}$} & \multirow[t]{3}{*}{ IC ( 95\%) } \\
\hline & \multicolumn{2}{|c|}{ Sim } & \multicolumn{2}{|c|}{ Não } & \multicolumn{2}{|c|}{ Total } & & \\
\hline & $\mathrm{n}$ & $\%$ & Não & $\%$ & $\mathrm{n}$ & $\%$ & & \\
\hline \multicolumn{9}{|l|}{$\begin{array}{l}\text { Utilização de serviços nos } \\
\text { últimos } 12 \text { meses }\end{array}$} \\
\hline \multicolumn{9}{|l|}{ Consulta ao médico } \\
\hline Sim & 72,165 & 72.20 & 64,322 & 45.67 & 136,487 & 56.68 & 3.09 & $3,04-3,14$ \\
\hline Não & 27,792 & 27.80 & 76,528 & 54.33 & 104,320 & 43.32 & & \\
\hline Total & 99,957 & 100.00 & 140,850 & 100.00 & 240,807 & 100.00 & & \\
\hline \multicolumn{9}{|l|}{ Internação } \\
\hline Sim (excluídos os partos) & 10,102 & 10.26 & 4,265 & 3.10 & 14,367 & 6.09 & 3.57 & $3,44-3,70$ \\
\hline Não & 88,383 & 89.74 & 133,125 & 96.90 & 221,508 & 93.91 & & \\
\hline Total & 98,485 & 100.00 & 137,390 & 100.00 & 235,875 & 100.00 & & \\
\hline \multicolumn{9}{|l|}{$\begin{array}{l}\text { Acesso a serviços de saúde } \\
\text { nas } 2 \text { últimas semanas }\end{array}$} \\
\hline \multicolumn{9}{|l|}{ Procura de atendimento } \\
\hline Sim & 21,214 & 21.22 & 12,463 & 8.85 & 33,677 & 13.98 & 2.78 & $2,71-2,84$ \\
\hline Não & 78,748 & 78.78 & 128,394 & 91.15 & 207,142 & 86.02 & & \\
\hline Total & 99,962 & 100.00 & 140,857 & 100.00 & 240,819 & 100.00 & & \\
\hline \multicolumn{9}{|l|}{ Atendimento realizado } \\
\hline Sim & 20,235 & 95.39 & 12,032 & 96.53 & 32,267 & 95.81 & 0.74 & $0,66-0,83$ \\
\hline Não & 979 & 4.61 & 432 & 3.47 & 1,411 & 4.19 & & \\
\hline Total & 21,214 & 100.00 & 12,464 & 100.00 & 33,678 & 100.00 & & \\
\hline \multicolumn{9}{|l|}{ Atendimento realizado através do SUS } \\
\hline $\operatorname{Sim}$ & 10,517 & 53.13 & 5,041 & 42.93 & 15,558 & 49.33 & 1.51 & $1,44-1,58$ \\
\hline Não & 9,276 & 46.87 & 6,702 & 57.07 & 15,978 & 50.67 & & \\
\hline Total & 19,793 & 100.00 & 11,743 & 100.00 & 31,536 & 100.00 & & \\
\hline \multicolumn{9}{|l|}{$\begin{array}{l}\text { Motivos selecionados da procura } \\
\text { de atendimento }\end{array}$} \\
\hline Acidente ou lesão & 744 & 3.87 & 775 & 8.56 & 1,519 & 5.37 & 0.45 & $0,41-0,50$ \\
\hline Tratamento ou reabilitação & 2,863 & 14.90 & 991 & 10.95 & 3,854 & 13.64 & 1.36 & $1,26-1,47$ \\
\hline Prevenção & 8,303 & 43.22 & 4,525 & 49.98 & 12,828 & 45.39 & 0.86 & $0,83-0,90$ \\
\hline Doença & 7,300 & 38.00 & 2,762 & 30.51 & 10,062 & 35.60 & 1.25 & $1,18-1,31$ \\
\hline Total & 19,210 & 100.00 & 9,053 & 100.00 & 28,263 & 100.00 & 1.00 & \\
\hline \multicolumn{9}{|l|}{$\begin{array}{l}\text { Tipo de serviço onde procurou } \\
\text { atendimento de saúde }\end{array}$} \\
\hline Atendimento ambulatorial & 13,577 & 67.66 & 7,407 & 70.75 & 20,984 & 68.72 & 0.96 & $0,92-0,99$ \\
\hline PS ou emergência e hospital & 5,769 & 28.75 & 2,788 & 26.63 & 8,557 & 28.02 & 1.08 & $1,03-1,14$ \\
\hline Farmácia & 410 & 2.04 & 157 & 1.50 & 567 & 1.86 & 1.36 & $1,13-1,65$ \\
\hline Laboratório ou clínica para exames & 191 & 0.95 & 83 & 0.79 & 274 & 0.90 & 1.20 & $0,92-1,57$ \\
\hline Atendimento domiciliar & 120 & 0.60 & 34 & 0.32 & 154 & 0.50 & 1.84 & $1,24-2,75$ \\
\hline Total & 20,067 & 100.00 & 10,469 & 100.00 & 30,536 & 100.00 & & \\
\hline
\end{tabular}

(continua) 
Tabela 3

(continuação)

\begin{tabular}{|c|c|c|c|c|c|c|c|c|}
\hline \multirow[t]{3}{*}{ Variáveis } & \multicolumn{6}{|c|}{ Declaração de Doença Crônica } & \multirow{3}{*}{$\begin{array}{l}\text { Odds } \\
\text { Ratio }\end{array}$} & \multirow[t]{3}{*}{ IC ( $95 \%)$} \\
\hline & \multicolumn{2}{|c|}{ Sim } & \multicolumn{2}{|c|}{ Não } & \multicolumn{2}{|c|}{ Total } & & \\
\hline & $\mathrm{n}$ & $\%$ & Não & $\%$ & $\mathrm{n}$ & $\%$ & & \\
\hline \multicolumn{9}{|l|}{ Motivo da não procura } \\
\hline Não houve necessidade & 71,499 & 90.81 & 125,559 & 97.80 & 197,058 & 95.14 & 0.93 & $0,92-0,94$ \\
\hline Não tinha dinheiro & 2,210 & 2.81 & 877 & 0.68 & 3,087 & 1.49 & 4.11 & $3,79-4,45$ \\
\hline Local distante ou de dificil acesso & 682 & 0.87 & 302 & 0.24 & 984 & 0.48 & 3.68 & $3,21-4,23$ \\
\hline Dificuldade de transporte & 361 & 0.46 & 122 & 0.10 & 483 & 0.23 & 4.82 & $3,91-5,96$ \\
\hline Horário incompatível & 871 & 1.11 & 479 & 0.37 & 1,350 & 0.65 & 2.96 & $2,65-3,32$ \\
\hline Atendimento demorado & 1,005 & 1.28 & 404 & 0.31 & 1,409 & 0.68 & 4.06 & $3,61-4,56$ \\
\hline Ausência de especialista compatível & 307 & 0.39 & 87 & 0.07 & 394 & 0.19 & 5.75 & $4,51-7,35$ \\
\hline Achou que não tinha direito & 21 & 0.03 & 9 & 0.01 & 30 & 0.01 & 3.8 & $1,66-8,95$ \\
\hline Não tinha quem o acompanhasse & 200 & 0.25 & 25 & 0.02 & 225 & 0.11 & 13.04 & $8,47-20,24$ \\
\hline Outro & 1,581 & 2.01 & 516 & 0.40 & 2,097 & 1.01 & & \\
\hline Total & 78,737 & 100.00 & 128,380 & 100.00 & 207,117 & 100.00 & & \\
\hline
\end{tabular}

Fonte : Fundação IBGE. Pesquisa Nacional por Amostra de Domicílios - PNAD.

Nota: Excluem os casos de resposta ignorada.

Cerca de 56,6\% dos entrevistados referiram pelo menos uma consulta médica nos últimos 12 meses. Dentre aqueles que referiram problemas crônicos de saúde, $72,2 \%$ (IC $95 \%$ : 72,1-72,2) utilizaram consultas médicas, enquanto entre os não portadores a taxa de utilização foi de 45,6\% (IC $95 \%$ : 45,6-45,7). Portanto, a probabilidade de ter utilizado pelo menos uma consulta médica nos últimos 12 meses foi 3,09 vezes maior entre os portadores de doenças crônicas.

O número médio de consultas médicas para os portadores de problemas crônicos foi de 5,0 (IC95\%: 4,9-5,1) e para os demais, 3,1 consultas (IC95\%: 3,1-3,2), apontando maior utilização de consultas entre os portadores de problemas crônicos, como seria esperado. A diferença foi de aproximadamente 2 consultas/ano. Os valores medianos, 3,0 e 2,0 consultas respectivamente, mostram a mesma tendência, porém apontam uma diferença menor, de apenas 1 consulta/ano.

Os portadores de problemas crônicos de saúde que possuem planos de saúde, particulares, empresariais, ou de órgãos públicos apresentaram maior consumo de consultas médicas nos últimos 12 meses. A média de consultas médicas foi de 5,6 (IC95\%: 5,5-5,7) para aqueles com plano de saúde e 4,7 (IC95\%: 4,6$4,7)$ para os demais. Embora a posse de plano de saúde tenha possibilitado maior consumo médio de consultas médicas, a diferença foi apenas de uma consulta, mostrando assim que a cobertura oferecida pelo Sistema Único de Saúde tem sido próxima àquela oferecida pela posse de um plano particular, empresarial, ou de órgão público.

Como já mencionado, um dos fatores condicionantes do consumo de serviços de saúde é a renda. No entanto, não foram observadas grandes variações no número médio de consultas $(4,7$ a 5,2) por faixa de renda, embora a diferença seja estatisticamente significante $(\mathrm{F}=6,22 ; \mathrm{p}=0,00)$. O menor número médio de consultas foi observado entre os indivíduos com renda familiar entre 2 e 3 salários-mínimos e os maiores valores nas classes extremas, isto é, para as pessoas sem rendimento ou com renda familiar acima de 10 salários-mínimos.

Em países desenvolvidos a taxa de utilização de consultas médicas é bem mais elevada. Em estudo conduzido por Izumi et al. (2001) no Japão, observou-se que cerca de $92 \%$ dos indivíduos adultos utilizam, em média, 2 a 2,5 consultas médicas/mês. Na Califórnia, entre os participantes adultos, maiores de 40 anos de idade, em um estudo de intervenção, Lorig et al. (2001) relatam cerca de 5,86 visitas ao médico ou a um serviço de emergência nos últimos 6 meses. No inquérito de saúde norte-americano observou-se, em 1995, média de 5,8 consultas médicas nos últimos 12 meses, para a população total. Esse valor aumenta com a idade chegando a 11,1 consultas para os indivíduos com 65 anos e mais. A taxa de utilização é maior entre as mulheres e entre os mais pobres (ren- 
da familiar abaixo de 15 mil dólares anuais) e praticamente igual entre brancos e negros; nas diferentes regiões do país.

Em comunidade de idosos de Porto Rico, Cordero et al. (2000) encontraram taxa de utilização semelhante às obtidas pela PNAD. Guerra et al. (2001) observaram entre os idosos da coorte de Bambuí, que o número de consultas médicas nos últimos 12 meses aumentava de acordo com a gravidade do estado de saúde. Entre os idosos que não tiveram nenhuma internação hospitalar em 12 meses, 62,8\% referiram 2 ou menos consultas médicas e $17,4 \%$ referiram 5 ou mais consultas, enquanto entre os idosos que apresentaram duas ou mais internações no período de um ano 11,6\% referiram 2 ou menos consultas e $58,9 \%$ referiram 5 ou mais.

Das pessoas entrevistadas, $6,1 \%$ referiram pelo menos uma internação hospitalar nos últimos 12 meses. A taxa entre os portadores de problemas crônicos foi de 10,3\% (IC95\%: 10,210,4 ) e entre os demais, 3,1\% (IC $95 \%$ : 3,0-3,2), denotando consumo três vezes e meia maior para o primeiro grupo. Para a população adulta do Japão (maiores de 39 anos), a taxa de internação hospitalar variou de 18 a 23\% dependendo do sexo e hábito de fumar (Izumi et al., 2001). Taxa semelhante foi observada entre os idosos de Porto Rico, 22,4\%, e na coorte de Bambuí, 22,6\% (Cordero et al., 2000 e Guer- ra et al., 2001). Os dados desses inquéritos não são comparáveis aos da PNAD, pois as faixas etárias da população estudada são diferentes. No caso da PNAD, a informação refere-se à população acima de 14 anos e, portanto, a taxa de internações deve ser menor. Além disso, com a implantação do Sistema Único de Saúde houve crescimento das consultas médicas e redução das internações hospitalares, durante a década de 1990, em todas as regiões brasileiras, exceto na região Norte, em decorrência do novo modelo assistencial (Vianna et al., 2001).

A figura 2 apresenta a distribuição da proporção de internações e da restrição das atividades cotidianas por motivo de saúde segundo nível de escolaridade. Ambas as distribuições apresentam gradiente inversamente proporcional ao nível de escolaridade, conforme o esperado. Os indivíduos com menos de 3 anos de escolaridade, analfabetos funcionais, apresentam as maiores taxas de internação e de restrição, $13,0 \%$ e 15,2\% respectivamente, enquanto os indivíduos com 11 ou mais anos de escolaridade apresentam as menores taxas de internação e restrição, respectivamente, $9.0 \%$ e $9,4 \%$, sendo $50 \%$ menor. A maior taxa de internação e de restrição das atividades entre os indivíduos com menor escolaridade pode estar relacionada a diferentes fatores. As piores condições de vida favorecem a ocorrência de doenças e dificultam a adoção de hábitos sa-

Figura 2

Taxa de internação e restrição de atividades de pessoas com doenças crônicas segundo escolariddade, Brasil, 1998.

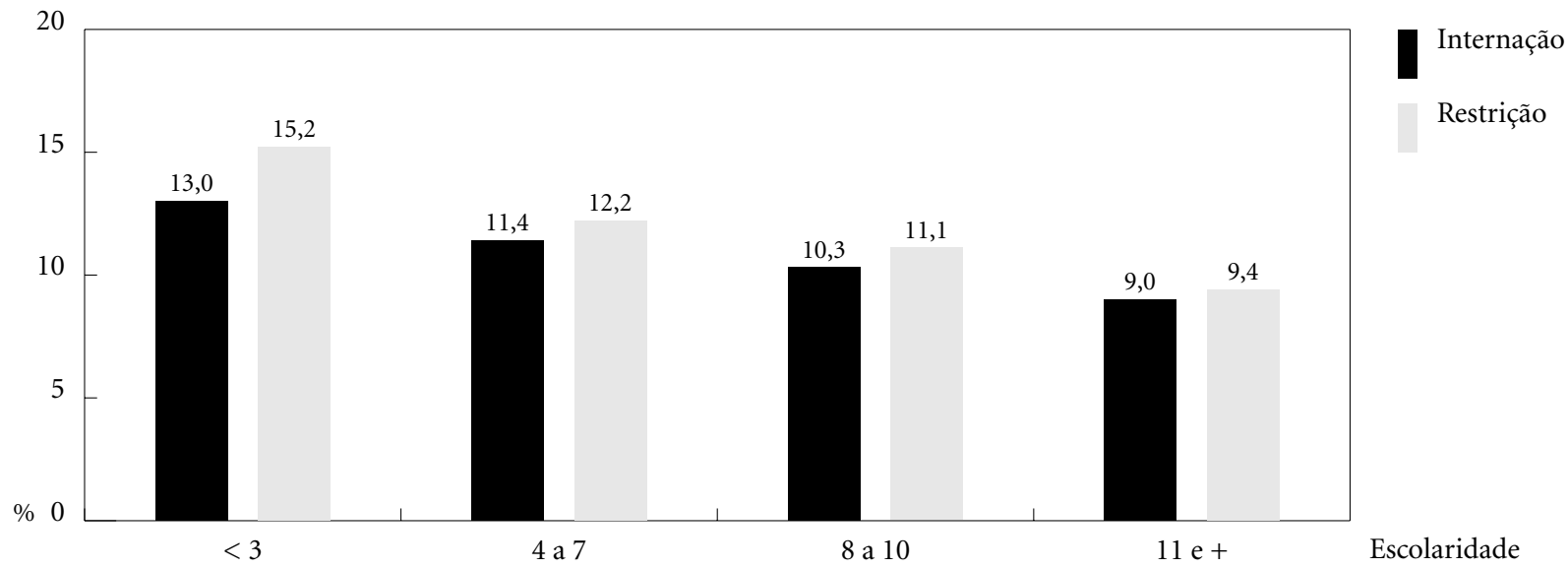


dios de vida. Além disso, entre os indivíduos com pior inserção social é mais freqüente que os diagnósticos sejam feitos em uma etapa mais avançada da doença, na qual podem ocorrer mais complicações. Finalmente, parte das internações hospitalares é determinada pelas precárias condições dos pacientes, o que os impossibilita de seguirem as prescrições médicas em regime ambulatorial. Todos esses fatores contribuem para aumentar, relativamente, a taxa de internação entre os indivíduos com menor escolaridade, na medida em que a escolaridade for tomada como proxi da inserção social. Mas há de se considerar ainda a ação direta da baixa escolaridade do indivíduo e as decorrentes dificuldades à compreensão das orientações médicas e de saúde.

A figura 3 mostra a taxa de internação segundo renda familiar e posse de plano de saúde particular, empresarial, ou de órgão público. A posse do plano de saúde passou de 7,5\% da população na faixa dos sem rendimentos para $83,7 \%$ na faixa de população com mais de 20 salários mínimos de renda familiar. As taxas de internações foram inversamente proporcionais ao nível de renda familiar. Entretanto, para todas as classes de rendimento, exceto a primeira, observaram-se maiores taxas de internações entre os indivíduos com posse de plano de saúde, sugerindo que houve maior fa- cilidade de internação para esses indivíduos quando comparados aos que não possuem.

Além do consumo de consultas médicas e internações hospitalares nos últimos 12 meses, foram avaliados vários aspectos da procura de serviços de saúde nos 15 dias anteriores à entrevista. Entre os indivíduos entrevistados, 14,0\% referiram ter procurado serviços de saúde nos últimos 15 dias. Entre os portadores de problemas crônicos de saúde, 21,2\% (IC95\%: 21,1-21,2) procuraram serviços de saúde nas duas últimas semanas, enquanto entre os demais, a taxa de procura foi de 8,8\% (IC95\%: 8,7-8,9). Assim, os portadores de doenças crônicas apresentaram 2,78 vezes mais procura por serviços de saúde.

Entre os indivíduos que procuraram serviços de saúde, 95,8\% receberam atendimento. Entre os portadores de problemas crônicos essa taxa foi de 95,4\% (IC95\%: 95,3-95,4), enquanto para os não portadores a taxa foi de 96,5\% (IC95\%: 96,4-96,5). Embora as taxas de atendimento tenham sido altas nos dois grupos, indicando a universalização da assistência, a proporção de não atendidos foi 35\% maior para os portadores de problemas crônicos de saúde.

Os atendimentos nas duas últimas semanas foram feitos pelo SUS para 49,3\% (IC95\%: 49,2-49,4) dos entrevistados. Para os porta-

Figura 3

Taxa de internação de pessoas com doenças crônicas nos últimos 12 meses, segundo renda familiar e posse de plano de saúde, Brasil, 1998.

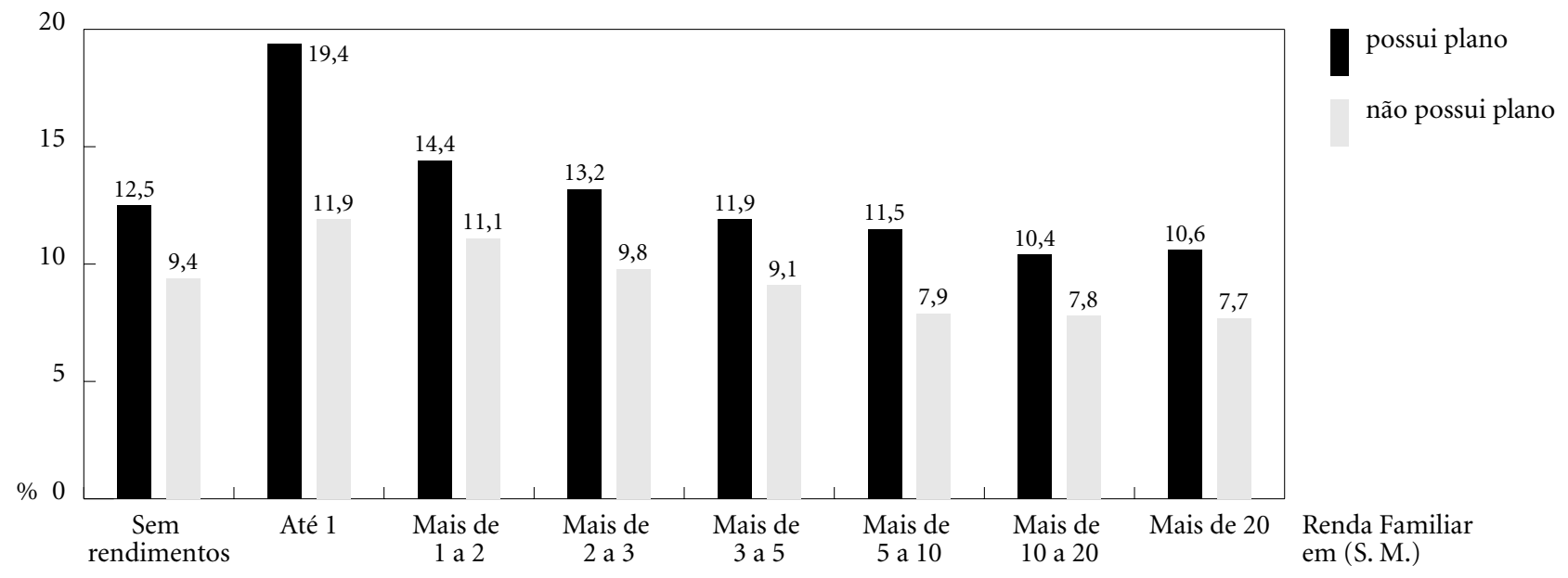


dores de problemas crônicos de saúde, 53,1\% (IC95\%: 52,9-53,3) dos atendimentos foram feitos pelo SUS. Para os não portadores, $42,9 \%$ (IC95\%: 42,8-43,0) dos atendimentos foram feitos pelo SUS. Portanto, mais atendimentos nas duas últimas semanas foram feitos pelo SUS para os portadores de doenças crônicas quando comparados aos indivíduos sem problemas crônicos de saúde.

Quanto aos motivos para a procura do atendimento, no grupo de portadores de problemas crônicos de saúde houve proporcionalmente menor procura por causa de acidentes ou lesões e maior procura por tratamento ou reabilitação e por doença do que entre os não portadores. Em ambos os grupos o motivo mais freqüentemente declarado foi prevenção, $43,2 \%$ e 50,0\% respectivamente. Entretanto, para o grupo dos portadores de doença crônica, a procura por tratamento, reabilitação ou por doença, quando somadas (52,9\%), ultrapassa a proporção da procura por prevenção.

Quanto ao tipo de serviço procurado, a distribuição foi relativamente semelhante para os dois grupos. O tipo de serviço mais procurado foi o atendimento ambulatorial, praticamente na mesma proporção (68 a 70\%) para os portadores e os não portadores de problemas crônicos de saúde. Os serviços de urgência e emergência apareceram em seguida com cerca de $28 \%$ da procura. Esses dados demonstram que a inversão entre a assistência hospitalar e a ambulatorial, pretendida pela implementação do SUS, está sendo concretizada em grande medida. Apenas $1 \%$ da procura foi por exames diagnósticos complementares, não havendo diferença entre os grupos, contrariando, em certa medida, a expectativa de maior utilização de exames complementares pelos portadores de problemas crônicos de saúde. Embora uma parcela muito reduzida da procura tenha sido destinada às farmácias, como locais para atendimento, os doentes crônicos lançaram mão desse recurso com mais freqüência (cerca de $36 \%$ a mais), possivelmente para fazer frente às necessidades não atendidas pelos demais serviços. Apenas 0,5\% dos atendimentos foram de tipo domiciliar, mas sua utilização foi 1,84 vezes mais freqüente entre os doentes crônicos.

Apenas 4,18\% (1.411) dos indivíduos procuraram serviços de saúde nos últimos 15 dias, mas não foram atendidos. Os principais motivos para o não atendimento foram: não conseguir vaga ou senha $(45,5 \%)$; não haver mé- dico atendendo (29,4\%); ausência de serviço ou profissional especializado $(6,0 \%)$; e espera muito demorada (5,5\%). Apenas $1,0 \%$ desses indivíduos afirmaram que não foi atendido porque não tinha dinheiro. Entre os portadores de doenças crônicas foi mais freqüente não ter sido atendido por demora excessiva na espera pelo atendimento ou por não conseguir vaga ou senha, quando comparado aos não portadores. As diferenças, entretanto, não foram estatisticamente significantes.

Entre os que informaram algum problema de saúde nos últimos 15 dias, 4,9\% não procuraram serviços de saúde. No grupo dos portadores de doenças crônicas essa proporção foi de $9,2 \%$ (IC95\%:9,1-9,3) enquanto nos demais foi de apenas 2,2\% (IC95\%: 2,1-2,2). Os motivos informados para não procurar serviços de saúde foram semelhantes nos dois grupos, porém as dificuldades relativas foram sempre maiores para os portadores de doenças crônicas. Os motivos informados com maior freqüência foram: a falta de dinheiro, a possível demora no atendimento e o horário inadequado de funcionamento dos serviços. Embora tenha sido mencionada com menor freqüência, a ausência de um acompanhante foi mais importante como motivo de não procura entre os portadores de doenças crônicas, reforçando, assim, a necessidade de atendimento domiciliar para uma parte, ainda que pequena, desses indivíduos.

De maneira geral, os dados da PNAD, aqui apresentados, indicam que a quase totalidade da população com algum problema de saúde procura e obtém atendimento em um serviço da saúde, prestado, em aproximadamente 49\% dos casos, pelo componente público do Sistema Único de Saúde.

As taxas de utilização, embora baixas quando comparadas àquelas observadas nos países desenvolvidos, refletem, em parte, a composição etária da população e as próprias características do modelo assistencial adotado.

\section{Conclusões}

A primeira conclusão relativa à análise efetuada diz respeito à consistência dos dados. Embora exista alguma restrição ao uso da morbidade referida em inquéritos populacionais, baseada na idéia de que essas informações são inespecíficas e pouco precisas, as comparações feitas entre a prevalência informada de doenças 
crônicas e a auto-avaliação do estado de saúde, a restrição das atividades cotidianas e ter estado acamado permitem concluir que essas informações podem ser utilizadas.

A segunda conclusão importante é que os dados da PNAD mostraram que os grupos com piores condições socioeconômicas apresentam maior prevalência, a despeito de concepções bastante divulgadas de que os problemas crônicos de saúde afetam igualmente os diferentes grupos sociais ou, ainda, de que os problemas crônicos de saúde são mais freqüentes entre as pessoas com melhores condições de vida porque elas tendem a ser mais velhas.

Conforme o esperado, os portadores de problemas crônicos de saúde consumiram mais consultas médicas e internações hospitalares nos últimos 12 meses do que os não portadores. O maior consumo de internações hospitalares entre os mais pobres, independentemente da posse de plano de saúde, sugere que a universalização do acesso é uma realidade. Evidentemente, os dados não permitem avaliar o acesso diferencial a serviços com diferentes graus de sofisticação tecnológica, medindo apenas o uso de serviços, em geral.

Outro aspecto do consumo de serviços que merece ser destacado é o uso preferencial de serviços ambulatoriais em comparação a serviços de urgência e emergência, farmácias e laboratórios, para problemas de saúde ocorridos nos 15 dias anteriores a entrevista. Esse dado é coerente com a mudança do modelo assistencial pretendida pelo SUS e também com as necessidades dos portadores de problemas crônicos de saúde.

Dado extremamente relevante é a baixa porcentagem de pessoas que buscaram atendimento e não foram atendidas ou tiveram um pro- blema de saúde e não buscaram atendimento por uma série de dificuldades de acesso. Claro está que, em termos absolutos, há cerca de 340 mil pessoas que se enquadram em uma dessas categorias, porém, cerca de 3,6 milhões de pessoas foram atendidas pelos serviços de saúde nos 15 dias que antecederam a entrevista.

Parcela importante desses atendimentos foi realizada em estabelecimentos que a população identificou como sendo serviços de saúde financiados pelo SUS. Tendo em vista a prática condenável ainda vigente em vários serviços conveniados ou contratados, de cobrança irregular de pagamentos diretos pelos pacientes mesmo que o serviço esteja sendo custeado pelo SUS, é de se supor que parcela ainda maior dos atendimentos tenha sido financiada pelo poder público.

Finalmente, é possível concluir que a utilização dos dados da PNAD pode ser ainda maior do que a que foi feita até aqui, visto que uma análise descritiva preliminar como a aqui apresentada mostrou-se tão rica em informações. Certamente a exploração das desigualdades regionais, a elaboração de modelos multivariados para identificar preditores de utilização de serviços, as comparações entre grupos etários, gênero, e muitas outras, poderiam ampliar ainda mais o conhecimento sobre os padrões de acesso e utilização de serviços de saúde pela população brasileira.

Para que isso possa ser feito é importante que os inquéritos nacionais tenham periodicidade regular, mantendo, na medida do possível, metodologias que permitam a comparação entre eles e que a divulgação dos dados seja amplamente facilitada aos pesquisadores interessados. 


\section{Referências bibliográficas}

Benyamini Y, Leventhal EA, Leventhal H 2000. Gender differences in processing information for making self-assessment of health. Psychosomatic Medicine 62:354-364.

Branch L 2000. Assessment of chronic care need and use. The Gerontologist 40(4):390-396.

Cordero BMR, Negrón CF, Vigo MDCP, Vázquez DA, Vázquez MO 2000. Diagnóstico de necesidades de salud de la población de adultos vejos de un sector de la comunidad de Puerto Novo. Puerto Rico Health Science Journal 19(2):139-144.

Cox ME, Asselin S, Gracovetsky SA, Richards MP, Newmann NM 2000. Relationship between functional evaluation measures and self-assessment in nonacute low back pain. Spine 25(14):1.817-1.826.

Elliot AM, Smith BH, Smith WC, Chambers WA 2000. Changes in chronic pain severity over time: the chronic pain grade as a valid measure. Pain 88(3):303-308.

Enns MW, Larsen DK, Cox BJ 2000. Discrepancies between self and observer ratings of depression. The relationship to demographic, clinical and personality variables. Journal of Affective Disorders 60:33-41.

Ferraro KF \& Su Y 2000. Physician-evaluated and self-reported morbidity for predicting disability. American Journal of Public Health 90(1):103-108.

Guerra HL, Firmo JOA, Uchoa E, Lima-Costa MFF 2001. The Bambuí health and aging study (BHAS): factors associated with hospitalization of the elderly. Cadernos de Saúde Pública 17(6):1.345-1.356.

Izumi Y et al. 2001. Impact of smoking habit on medical care use and its costs: a prospective observation of
National Health Insurance beneficiaries in Japan. International Journal of Epidemiology 30:616-621.

Lorig KR et al. 2001. Chronic disease self-management program. Two-year health status and health care utilization outcome. Medical Care 39(11):1.217-1.223.

Rathouz PJ et al. 1998. Short-term consistency in self-reported physical functioning among elderly women. American Journal of Epidemiology 147(8):764-773.

Rauber C 1999. Disease management can be good for what ails patients and insurers. Modern Healthcare March 29:48-54.

Sen A 2002. Health: perception versus observation. Editorial BMJ 324:860-861. (13 April).

Trevena LJ, Nutbeam D, Simpson JM 2001. Asking the right questions of disadvantaged and homeless communities: the role of housing, patterns of illness and reporting behaviours in the measurement of health status. Australian and New Zeland Journal of Public Health 25(4):298-304.

US Department of Health and Human Services 1998. Health, United States, with socioeconomic status and health chartbook, pp. 287-288.

Vianna SM, Nunes A, Santos JRS, Barata RB 2001. Medindo a desigualdade em saúde no Brasil: uma proposta de monitoramento. OPS/IPEA, Brasília.

Wu SC, Li CY, Ke DS 2000. The agreement between selfreporting and clinical diagnosis for selected medical conditions among elderly in Taiwan. Public Health 114:137-142.

Trabalho apresentado em 8/8/2002 Aprovado em 11/9/2002

Versão final apresentada em 23/9/2002 